\title{
Prognostic Importance of the Cause of Renal Failure in Patients With Cirrhosis
}

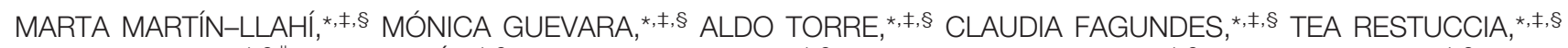

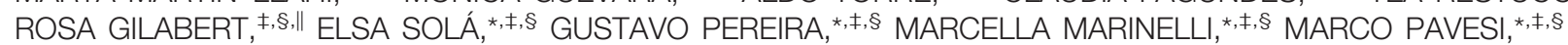 \\ JAVIER FERNÁNDEZ, ${ }^{*, \neq, \S}$ JUAN RODÉS, ${ }^{*, \neq, \S}$ VICENTE ARROYO, ${ }^{*, \neq, \S}$ and PERE GINÈS ${ }^{*, \neq, \S}$ \\ *Liver Unit, Hospital Clínic, University of Barcelona, Barcelona, Spain; 'Institut d'Investigacions Biomèdiques August-Pi-Sunyer (IDIBAPS), Barcelona, Spain; ${ }^{2}$ Centro \\ de Investigación Biomédica en Red de Enfermedades Hepáticas y Digestivas (CIBEREHD), Barcelona, Spain; and "Radiology Department, Hospital Clínic, University \\ of Barcelona, Barcelona, Spain
}

BACKGROUND \& AIMS: The prognostic value of the different causes of renal failure in cirrhosis is not well established. This study investigated the predictive value of the cause of renal failure in cirrhosis. METHODS: Five hundred sixty-two consecutive patients with cirrhosis and renal failure (as defined by serum creatinine $>1.5$ $\mathrm{mg} / \mathrm{dL}$ on 2 successive determinations within 48 hours) hospitalized over a 6-year period in a single institution were included in a prospective study. The cause of renal failure was classified into 4 groups: renal failure associated with bacterial infections, renal failure associated with volume depletion, hepatorenal syndrome (HRS), and parenchymal nephropathy. The primary end point was survival at 3 months. RESULTS: Four hundred sixty-three patients $(82.4 \%)$ had renal failure that could be classified in 1 of 4 groups. The most frequent was renal failure associated with infections (213 cases; 46\%), followed by hypovolemia-associated renal failure (149; $32 \%)$, HRS (60; $13 \%)$, and parenchymal nephropathy (41; $9 \%)$. The remaining patients had a combination of causes or miscellaneous conditions. Prognosis was markedly different according to cause of renal failure, 3-month probability of survival being $73 \%$ for parenchymal nephropathy, $46 \%$ for hypovolemia-associated renal failure, $31 \%$ for renal failure associated with infections, and $15 \%$ for HRS $(P<.0005)$. In a multivariate analysis adjusted for potentially confounding variables, cause of renal failure was independently associated with prognosis, together with MELD score, serum sodium, and hepatic encephalopathy at time of diagnosis of renal failure. CONCLUSIONS: A simple classification of patients with cirrhosis according to cause of renal failure is useful in assessment of prognosis and may help in decision making in liver transplantation.

Keywords: Cirrhosis; Model for End-Stage Liver Disease Score; MELD; Hepatorenal Syndrome.

$\mathrm{P}$ atients with cirrhosis may develop renal failure because of a variety of causes, including bacterial infections, volume depletion caused by bleeding or fluid losses, intrinsic renal diseases, mainly glomerulonephri- ties associated with alcoholic liver disease or hepatitis B or $\mathrm{C}$ infection or other chronic kidney diseases, and nephrotoxicity, particularly related to treatment with nonsteroidal anti-inflammatory drugs (NSAIDs). ${ }^{1-3}$ It has been known for many years that patients with cirrhosis and renal failure have a reduced survival than patients without renal failure. ${ }^{1-3}$ This explains, at least in part, the power of the Model for End-Stage Liver Disease (MELD) score in the assessment of prognosis in cirrhosis, because in addition to parameters of liver function it includes serum creatinine. ${ }^{4}$ Compared with the abundance of studies that show the prognostic value of renal function parameters, particularly serum creatinine (reviewed in ${ }^{1-3}$ ), it is not known whether the cause of renal failure is relevant to prognosis. This information may be important not only for clinical management of patients and classification of patients in therapeutic trials but also in decision making in liver transplantation. On this background, we designed a prospective, single-center study to evaluate the causes of renal failure in hospitalized patients with cirrhosis and their relevance to prognosis.

\section{Materials and Methods Study Population}

All consecutive patients with cirrhosis and renal failure hospitalized at the Liver Unit of the Hospital Clínic of Barcelona from January 2002 to September 2008 were included in a prospective study aimed at investigating the causes of renal failure in cirrhosis and their relationship with prognosis. Patients admitted for complications of cirrhosis as well as for conditions unrelated to liver disease that had renal failure at admission or developed renal failure during hospitalization were included. The only patients excluded from the study were (1) patients previously treated with liver transplantation,

Abbreviations used in this paper: HRS, hepatorenal syndrome; MELD, Model for End-Stage Liver Disease; NSAID, nonsteroidal antiinflammatory drug.

(C) 2010 by the AGA Institute

$0016-5085 / \$ 36.00$

doi:10.1053/j.gastro.2010.07.043 
(2) patients with chronic renal failure who were under hemodialysis treatment before admission, and (3) patients with cirrhosis and previous kidney transplantation.

\section{Study Design and Interventions}

All patients had their renal function assessed at admission and at regular intervals throughout hospitalization. If renal failure (see "Definitions") was present at admission or developed during hospitalization, the following work-up for identification of the cause of renal failure was performed: (1) Systematic medical history, with particular emphasis on factors that could be associated with volume depletion (gastrointestinal bleeding, excessive weight loss in patients with ascites treated with diuretics, gastrointestinal fluid losses), signs of bacterial infection, or therapy with potentially nephrotoxic drugs, particularly NSAIDs; (2) complete physical examination; (3) laboratory tests, including liver tests, serum electrolytes, cell blood count, urinalysis, and examination of ascitic fluid; (4) cultures of blood, urine, and ascitic fluid and cultures of other organic fluids, when clinically indicated; and (5) renal ultrasonography. Laboratory tests, ascitic fluid analysis, urinalysis, and cultures were performed within 24 hours of the diagnosis of renal failure, whereas renal ultrasonography was performed within a time frame of 3 days unless there was suspicion of obstructive renal disease, in which case it was performed within the same day.

Management of renal failure was done according to the following scheme: (1) in all patients receiving diuretics or other drugs that could induce renal failure, the drugs were withdrawn immediately after the diagnosis of renal failure; (2) in patients with signs suggestive of volume depletion, plasma expanders were administered, and specific therapy was given according to the underlying cause of volume depletion (ie, pharmacologic or endoscopic therapy or both in patients with variceal bleeding); patients with hypovolemic shock were treated with plasma expansion and blood transfusion; (3) patients with suspected bacterial infection were treated with empiric antibiotic therapy according to previously established protocols ${ }^{5,6}$; in patients with septic shock vasoactive drugs and plasma expansion were given in addition to antibiotics; (4) patients with hepatorenal syndrome (HRS) with serum creatinine greater than $2 \mathrm{mg} / \mathrm{dL}$ were treated with terlipressin and albumin, as described elsewhere ${ }^{1}$; and (5) renal replacement therapy was used for patients with severe volume overload, metabolic acidosis, or hyperkalemia unresponsive to medical therapy. Patients in terminal condition who were not candidates for liver transplantation were not treated with specific therapies (ie, terlipressin) or renal replacement therapy. Management of complications of cirrhosis was done with the guidelines described in detail elsewhere. ${ }^{5}$ Patients with spontaneous bacterial peritonitis were treated with albumin together with antibiotics at the time of diagnosis of the infection to prevent the development of renal failure..$^{1,7}$ After discharge from the hospital, patients were followed up for at least 3 months in the outpatient clinic.

\section{Definitions}

Cirrhosis. The diagnosis of cirrhosis was based on liver biopsy, when available, or on a combination of clinical, biochemical, ultrasonographic, and endoscopic findings.

Renal failure. Renal failure was diagnosed when serum creatinine concentration was greater than 1.5 $\mathrm{mg} / \mathrm{dL}(133 \mu \mathrm{mol} / \mathrm{L})$ in 2 consecutive measurements performed within a 48 -hour period. This value of serum creatinine was used because it has been selected in several consensus conferences as a cutoff to define renal failure in cirrhosis. ${ }^{8,9}$

Causes of renal failure. The following classification, based on clinical and analytical findings, was used to classify patients with cirrhosis and renal failure into 4 different categories: (1) Renal failure associated with infections. Renal failure was considered secondary to an infection when patients had an ongoing infection in the absence of other causes of renal failure. The following definitions of specific infections were used: spontaneous bacterial peritonitis was defined as the presence of a polymorphonuclear count greater than 250 per $\mathrm{mm}^{3}$ in ascitic fluid in the absence of a source of infection in the peritoneal cavity ${ }^{10}$; secondary bacterial peritonitis was defined as a polymorphonuclear count greater than 250 per $\mathrm{mm}^{3}$ in ascitic fluid in the presence of inflammation or perforation of an abdominal organ ${ }^{10}$; spontaneous bacteremia was defined in the presence of positive blood cultures without an evident source of infection; pneumonia, urinary tract infection, cellulitis, biliary tract infection, gastroenteritis, and meningitis were defined with standard diagnostic criteria; finally, culture-negative sepsis was defined as the presence of fever $\left(>38^{\circ} \mathrm{C}\right)$, leukocytosis, or band forms together with negative cultures, requiring antibiotic therapy, after exclusion of conditions other than infection that could be responsible for the systemic inflammatory response. (2) Hypovolemia-related renal failure. Renal failure was considered related to hypovolemia when patients had a history of fluid losses in the preceding days (because of bleeding, diuretics, or other causes, mainly gastrointestinal fluid losses) together with compatible findings and the absence of other causes of renal failure. (3) Renal failure because of parenchymal nephropathy was considered when there was either proteinuria greater than $500 \mathrm{mg} / 24$ hours, abnormal urine sediment with more than 50 red cells per highpower field, or abnormal renal ultrasound findings in the absence of other causes of renal failure; a renal biopsy was performed in these patients when there were no marked coagulation abnormalities (prothrombin time $>50 \%$ and platelet count $>50,000 / \mu \mathrm{L})$, and patients gave informed consent. (4) Hepatorenal syndrome. The 
diagnosis of HRS was made after exclusion of other potential causes of renal failure as follows: absence of signs of infection, hypovolemia or shock, no previous treatment with potentially nephrotoxic drugs, proteinuria $<500 \mathrm{mg} / \mathrm{d}$, hematuria $<50$ cells per high-power field, and no improvement of renal function (reduction in serum creatinine $<1.5 \mathrm{mg} / \mathrm{dL}$ ) after volume expansion. ${ }^{8}$ These criteria were used instead of the most recent criteria of the International Ascites Club because the latter were published when the inclusion of patients in the current study was almost finished. The assignment of patients into the 4 different categories was done when the results of all tests and ultrasonography were available, usually within a period of 5 days after diagnosis of renal failure. All cases were reviewed by 2 of the investigators, and an agreement was required for the classification of patients into 1 of the 4 categories. Disagreements were reconciled with the help of a third investigator. Patients who could not be classified in a specific category because of combination of findings of infection, hypovolemia, or parenchymal nephropathy were classified into a separate group of mixed causes.

\section{Statistical Analysis}

Univariate analyses of the variables associated with 3-month mortality or causes of renal failure were carried out by Student $t$ tests or 1-way analyses of variance (for continuous variables) and $\chi^{2}$ tests (for categorical variables). All variables analyzed for their relationship with survival or renal failure, including MELD score, were those obtained at the time of diagnosis of renal failure. Survival curves were estimated by Kaplan-Meier method.

Because all patients were followed for 3 months, a multivariate logistic regression model was fitted to estimate the effect of causes of renal failure on 3-month survival. Those variables associated with both 3 -month survival and causes of renal failure were selected as potential confounders. First, a logistic regression model, including causes of renal failure as the only factor, was fitted. Each potential confounder was then added into the model to get an adjusted estimate of the main effect studied. When the change in the estimated coefficient for the cause of renal failure was higher than $10 \%$, the confounding variable was kept in the model, otherwise it was dropped. The predictive ability of the fitted explanatory model was assessed by estimating the corresponding area under the receiver-operated curve (C-statistics) and its $95 \%$ confidence interval.

On the basis of the final logistic regression models, prognostic formulae were derived to estimate the probability of death at 3 months for the different individual patterns. The logit link is as follows: $\mathrm{P}($ event $)=\mathrm{ex}-$ $\mathrm{p}($ LOGIT $) / 1+\exp ($ LOGIT). Individual LOGIT values can be estimated for every combination of the variables in the model by the linear equation: LOGIT $=\alpha+\beta_{1} \mathrm{X}_{1}+$ $\beta_{2} \mathrm{X}_{2}+\ldots \beta_{\mathrm{n}} \mathrm{X}_{\mathrm{n}}$, where $\alpha$ is the constant term and the $\beta$ s are the estimated coefficients for the different model variables. The equation can be used to estimate the relationship between the different values of a specific model variable and the corresponding estimates for LOGIT (and probability of the outcome), simply by fixing the values of the rest of variables in the model.

The study was approved by the Institutional Review Board. Statistical analysis was performed with SPSS 15 for Windows (SPSS Inc, Chicago, IL). Results are expressed as mean \pm SD or counts and percentages, with $P<.05$ considered as statistically significant.

\section{Results}

\section{Causes of Renal Failure: Prevalence and Characteristics of Patients}

Five hundred sixty-two consecutive hospitalized patients with cirrhosis and renal failure were identified during the study period. Four hundred sixty-three patients $(82.4 \%)$ were classified into the 4 prespecified categories of renal failure. The most frequent cause was renal failure associated with infections (213 patients; $46 \%$ ), followed by hypovolemia-related renal failure (149; $32 \%)$, HRS (60,13\%), and parenchymal nephropathy (41, $9 \%)$. The remaining patients could not be classified into 1 of the 4 groups either because they had a mixed cause of renal failure (45 cases), drug-induced renal failure (42 cases; in most patients related to the administration of NSAIDs), or miscellaneous diseases (12 cases). Further information on the specific causes of renal failure in these 3 subsets of patients as well as their survival is given in the Supplementary Materials and Methods. Threemonth probability of survival of patients with druginduced or miscellaneous causes of renal failure was greater than $60 \%$, compared with less than $40 \%$ in patients with mixed cause of renal failure $(P=.009$; Supplementary Figure 1). These patients were not considered for further analysis. The disposition of all patients included in the study is shown in Supplementary Figure 2.

Among patients with renal failure associated with infections, the most frequent cause of infection was spontaneous bacterial peritonitis (32.9\%), followed by respiratory tract infections $(20.7 \%)$, spontaneous bacteremia $(11 \%)$, urinary tract infections $(10.3 \%)$, skin infections (8\%), and miscellaneous infections (17.4\%). One hundred forty-six patients $(70.5 \%)$ had positive microbiologic cultures. Gram-positive isolates were more frequent than gram-negative isolates ( $35.6 \%$ vs $32.2 \%$, respectively). Among patients with hypovolemia-related renal failure, the most frequent cause of volume depletion was gastrointestinal bleeding (42.3\%), followed by diuretics (30.2\%), bleeding from nongastrointestinal sources (8.7\%), gastrointestinal fluid losses (7.4\%), and miscellaneous circumstances (11.4\%). The presence of shock was common in patients with infections and hypovolemia-related renal failure. In patients with infections, $72(33.8 \%)$ of the 213 patients had shock, whereas shock was present in 26 
(17.4\%) of the 149 patients with hypovolemia. Among patients with HRS, 38 (63.3\%) had type 1 HRS and 22 (36.7\%) had type 2 HRS. In the group of patients with renal failure due to parenchymal nephropathy, more than half of the patients (24 cases; 58.5\%) had histologic confirmation of kidney disease. Ten patients had immunoglobulin A nephropathy, 9 had hepatitis C virus-associated nephropathy, and 5 had nephroangiosclerosis. A kidney biopsy could not be obtained in the remaining 17 patients.

Of the 285 patients who had a value of serum creatinine available within a time frame of 3 months before admission, 68 patients already had renal failure (serum creatinine $>1.5 \mathrm{mg} / \mathrm{dL}$ ). Of these patients, 31 developed superimposed acute impairment of renal function (as defined by an increase of $\geq 50 \%$ between the last preadmission value and the peak value of serum creatinine during hospitalization). Serum creatinine concentration in these patients increased from $2.0 \pm 0.8$ to $4.9 \pm 2.4$ $\mathrm{mg} / \mathrm{dL}$, respectively $(P<.001)$, whereas in patients without superimposed acute impairment of renal function corresponding values of serum creatinine were $2.1 \pm 0.6$ and $2.5 \pm 0.8 \mathrm{mg} / \mathrm{dL}$, respectively $(P<.001)$. In the 217 patients without renal failure before admission, serum creatinine concentration increased from $1.1 \pm 0.3$ to $2.9 \pm 1.4 \mathrm{mg} / \mathrm{dL}(P<.001)$. The survival of the 285 patients with an available serum creatinine concentration within 3 months before admission was divided into the following 3 groups: chronic renal failure, chronic renal failure with superimposed acute renal impairment, and acute renal failure (Supplementary Figure 3). Threemonth probability of survival was significantly better in patients with chronic stable renal failure than in patients with acute or acute-on-chronic renal failure $(P=.035)$. The distribution of patients of the 4 categories of renal failure according to the state of renal function before admission is shown in Supplementary Table 1.

The clinical characteristics and liver and renal function tests of the 4 groups of patients classified according to the cause of renal failure are shown in Table 1. Patients with renal failure due to parenchymal nephropathy were more frequently alcoholic, had higher arterial pressure, less severe liver failure, more marked impairment of renal function, and lower MELD score than patients from the other 3 groups. The mean values of peak serum creatinine concentrations during hospitalization were $3.4 \pm$ 1.6, $2.8 \pm 1.6,3.7 \pm 1.9$, and $3.8 \pm 2 \mathrm{mg} / \mathrm{dL}$ in patients with infections, hypovolemia, HRS, and parenchymal ne-

Table 1. Demographic and Clinical Characteristics, Liver Tests, and Renal Function at Diagnosis of Renal Failure in Patients Classified According to the Cause of Renal Failure

\begin{tabular}{|c|c|c|c|c|}
\hline & $\begin{array}{l}\text { Bacterial infections } \\
\qquad(n=213)\end{array}$ & $\begin{array}{l}\text { Hypovolemia } \\
(\mathrm{n}=149)\end{array}$ & $\begin{array}{l}\text { Hepatorenal syndrome } \\
\qquad(n=60)\end{array}$ & $\begin{array}{l}\text { Parenchymal nephropathy } \\
\qquad(\mathrm{n}=41)\end{array}$ \\
\hline Age, $y$, mean \pm standard deviation & $60 \pm 12$ & $64 \pm 13$ & $60 \pm 12$ & $63 \pm 11$ \\
\hline Sex, male, $\mathrm{n}(\%)$ & $147(69)$ & $104(69.8)$ & $46(76.7)$ & $30(73.2)$ \\
\hline Cause of cirrhosis (alcohol/HCV/other), $n$ & $87 / 69 / 57$ & $54 / 56 / 39$ & $16 / 24 / 20$ & $18 / 14 / 9$ \\
\hline Ascites, n (\%) & $185(86.9)$ & $107(71.8)$ & $60(100)$ & $31(75.6)$ \\
\hline Hepatic encephalopathy, n (\%) & $120(56.3)$ & $72(48.3)$ & $31(51.7)$ & $11(26.8)$ \\
\hline $\begin{array}{l}\text { Mean arterial pressure, } \mathrm{mm} \mathrm{Hg} \text {, mean } \pm \text { standard } \\
\text { deviation }\end{array}$ & $72 \pm 14$ & $76 \pm 13$ & $76 \pm 10$ & $91 \pm 13$ \\
\hline $\begin{array}{l}\text { Heart rate, beats per minute, mean } \pm \text { standard } \\
\text { deviation }\end{array}$ & $87 \pm 14$ & $84 \pm 16$ & $80 \pm 13$ & $77 \pm 11$ \\
\hline Serum albumin, $g / L$, mean \pm standard deviation & $27 \pm 5$ & $28 \pm 6$ & $28 \pm 5$ & $30 \pm 5$ \\
\hline $\begin{array}{l}\text { Serum bilirubin, } m g / d L \text {, mean } \pm \text { standard } \\
\text { deviation }\end{array}$ & $10.6 \pm 11.6$ & $4.6 \pm 6.8$ & $11.3 \pm 11.7$ & $1.6 \pm 1.4$ \\
\hline Prothrombin time, mean \pm standard deviation & $46 \pm 18$ & $58 \pm 19$ & $46 \pm 18$ & $67 \pm 17$ \\
\hline Child-Pugh A/B/C, n & $6 / 53 / 119$ & $16 / 53 / 61$ & $2 / 15 / 36$ & $8 / 23 / 5$ \\
\hline MELD score, mean \pm standard deviation & $24 \pm 7$ & $21 \pm 6$ & $26 \pm 8$ & $19 \pm 5$ \\
\hline $\begin{array}{l}\text { Serum creatinine at diagnosis, } m g / d L \text {, mean } \pm \\
\text { standard deviation }\end{array}$ & $2.4 \pm 1$ & $2.3 \pm 0.9$ & $2.3 \pm 0.7$ & $3 \pm 1.4$ \\
\hline $\begin{array}{l}\text { Serum sodium, } m E q / L \text {, mean } \pm \text { standard } \\
\text { deviation }\end{array}$ & $128 \pm 7$ & $130 \pm 7$ & $126 \pm 7$ & $135 \pm 4$ \\
\hline Hyponatremia, n (\%) & $123(58)$ & $62(42)$ & $42(70)$ & $4(10)$ \\
\hline $\begin{array}{l}\text { Glomerular filtration rate, } \mathrm{mL} / \mathrm{min} \text {, mean } \pm \\
\text { standard deviation }{ }^{b}\end{array}$ & $31 \pm 11$ & $32 \pm 11$ & $32 \pm 9$ & $25 \pm 10$ \\
\hline Urine sodium, $m E q / L$, mean \pm standard deviation $^{c}$ & $20 \pm 20$ & $27 \pm 28$ & $14 \pm 16$ & $30 \pm 22$ \\
\hline Urine volume, $m L / d$, mean \pm standard deviation ${ }^{d}$ & $874 \pm 710$ & $982 \pm 697$ & $733 \pm 408$ & $969 \pm 654$ \\
\hline
\end{tabular}

NOTE. HCV, hepatitis C virus.

${ }^{a}$ Defined as serum sodium lower than $130 \mathrm{mEq} / \mathrm{L}$.

${ }^{b}$ Estimated using the MDRD formula (Levey et $\mathrm{al}^{11}$ ).

${ }^{c}$ Not available in 82 patients with bacterial infections, 65 patients with hypovolemia, 15 patients with HRS, and 5 patients with parenchymal nephropathy.

${ }^{d}$ Not available in 36 patients with bacterial infections, 27 patients with hypovolemia, 7 patients with HRS, and 5 patients with parenchymal nephropathy. 
phropathy, respectively. Improvement of renal function (defined as a reduction in serum creatinine of at least 30\% of the maximum value of serum creatinine concentration during the hospitalization period) was observed in 117 (54.9\%) patients with infections, 88 (59.1\%) with hypovolemia-related renal failure, $18(30 \%)$ with HRS, and 9 (22\%) with parenchymal nephropathy.

\section{Survival}

At the end of the 3-month follow-up period, 151 (32.6\%) of the 463 patients were alive, 276 (59.6\%) had died, $21(4.6 \%)$ had received a transplant, and only 15 (3.2\%) were lost to follow-up. Overall, the 3 -month probability of survival was low, $38 \%$, with a median survival of only 41 days. Figure 1 shows the probability of survival of patients classified into the 4 different categories according to the cause of renal failure. Patients with parenchymal nephropathy had the best survival of the 4 categories (73\% probability of survival at 3 months), followed by patients with hypovolemia-related renal failure, who had a 3 -month probability of survival of $46 \%$. Among patients with hypovolemia-related renal failure, survival was better for those in whom diuretics were the cause of renal failure compared with those with bleeding (52\% vs $37 \%$ probability of survival at 3 months; $P<.05$ ). Patients with renal failure associated with infections and those with HRS had the lowest 3-month probability of survival of the 4 categories, $31 \%$ and $15 \%$, respectively. The survival of patients with renal failure associated with infections was low even when patients with septic shock were excluded (3-month probability of survival of patients with and without septic shock was $24 \%$ and $35 \%$, respec-

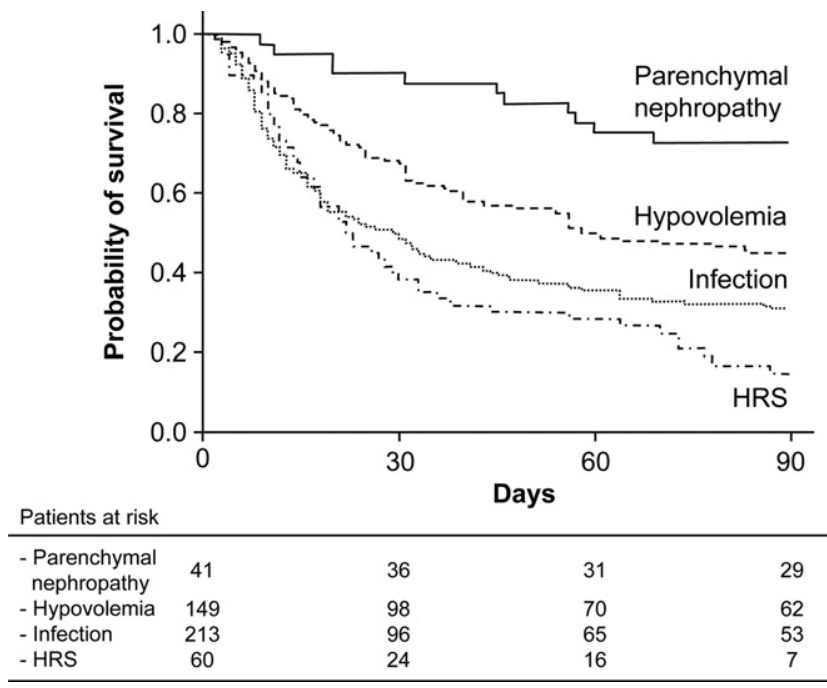

Figure 1. Three-month probability of survival of patients with cirrhosis and renal failure categorized in 4 different groups according to the cause of renal failure: renal failure due to parenchymal nephropathy, hypovolemia-associated renal failure, infection-associated renal failure, and HRS $(P<.0005)$. HRS was defined according to the classical diagnostic criteria (Arroyo et $a^{8}$ ). Figures under the curves are patients at risk at different time points. tively). Among patients with infection, those with respiratory tract infection and sepsis of unknown origin had the worst survival probability (14\% and $22 \%$, respectively), whereas those with urinary tract infections and spontaneous bacterial peritonitis had better survival $(37 \%$ and $32 \%$, respectively). The relationship between variables obtained at diagnosis of renal failure and 3-month survival is shown in Table 2.

To assess the relevance of the cause of renal failure on prognosis, an explanatory logistic regression model was performed. Potentially confounding variables included in the model were those associated with both 3-month survival and cause of renal failure. The final model included the cause of renal failure together with MELD score, serum sodium concentration, and the presence of hepatic encephalopathy at diagnosis of renal failure (Table 3).

Taking into account that the new diagnostic criteria of HRS $^{9}$ include patients with active bacterial infection, with the exception of those with septic shock, a second logistic regression analysis was performed with the patients classified according to 3 categories: (1) HRS with the use of the new definition ${ }^{9}$ (this group was composed of patients from 2 of the former categories: HRS and renal failure associated with infections, but excluding patients with septic shock; 201 patients were included in this new category); (2) hypovolemia-related renal failure; and (3) renal failure due to parenchymal nephropathy. Figure 2 shows the survival curves of patients divided according to these 3 categories of renal failure. The final logistic regression model included the cause of renal failure together with MELD score and serum sodium concentration at diagnosis of renal failure (Table 4). The areas under the receiver-operated curves of the 2 models were similar, 0.788 (95\% CI, 0.75-0.83) and 0.763 (95\% CI, 0.72-0.81), respectively. The area under the receiveroperated curve of a model with MELD score alone was 0.736 (95\% CI, $0.69-0.78$ ).

To analyze the relationship between MELD score and survival for each category of renal failure, prognostic formulae were constructed so that the importance of the cause of renal failure in terms of MELD points could be assessed (Supplementary Tables 2 and 3). In the model with 4 categories of renal failure, for patients with a similar probability of death, the differences in MELD scores with respect to parenchymal nephropathy were an average of $-7,-8$, and -16 MELD points for hypovolemia-related renal failure associated with infection, and HRS groups, respectively (Supplementary Materials and Methods and Supplementary Tables 4 and 5). In the model with 3 categories, the differences between parenchymal nephropathy and hypovolemia-related renal failure and HRS were an average of -7 and -10 MELD points, respectively (Supplementary Materials and Methods and Supplementary Table 6). A plot of the relationship between the probability of death at 3 months and 
Table 2. Relationship of Variables Obtained at Diagnosis of Renal Failure and 3-Month Mortality

\begin{tabular}{|c|c|c|c|}
\hline Variable & Alive & Dead & $P$ \\
\hline Age, $y$, mean \pm standard deviation & $62 \pm 13$ & $62 \pm 12$ & NS \\
\hline Sex, male, $\mathrm{n}(\%)$ & $113(75)$ & $190(69)$ & NS \\
\hline Cause of cirrhosis (alcohol/HCV/other), $n$ & $69 / 51 / 31$ & $94 / 97 / 85$ & NS \\
\hline Ascites, (\%) & $104(69)$ & $248(90)$ & $<.0005$ \\
\hline Hepatic encephalopathy, n (\%) & $53(35)$ & $166(60)$ & $<.0001$ \\
\hline Mean arterial pressure, $\mathrm{mm} \mathrm{Hg}$, mean \pm standard deviation & $80 \pm 15$ & $74 \pm 13$ & $<.0005$ \\
\hline Heart rate, beats per minute, mean \pm standard deviation & $82 \pm 14$ & $86 \pm 14$ & .006 \\
\hline Serum albumin, $g / L$, mean \pm standard deviation & $29 \pm 5$ & $26 \pm 5$ & $<.0005$ \\
\hline Serum bilirubin, $m g / d L$, mean \pm standard deviation & $3.0 \pm 3.9$ & $10.7 \pm 11.6$ & $<.0005$ \\
\hline Prothrombin time, $\%$, mean \pm standard deviation & $61 \pm 17$ & $47 \pm 19$ & $<.0005$ \\
\hline Child-Pugh A/B/C, $n$ & $26 / 69 / 41$ & $4 / 65 / 164$ & $<.0005$ \\
\hline MELD score, mean \pm standard deviation & $20 \pm 5$ & $26 \pm 8$ & $<.0005$ \\
\hline Serum creatinine at diagnosis, $m g / d L$, mean \pm standard deviation & $2.4 \pm 1$ & $2.4 \pm 1$ & NS \\
\hline Serum sodium, $m E q / L$, mean \pm standard deviation & $132 \pm 7$ & $128 \pm 7$ & $<.0005$ \\
\hline Hyponatremia, n (\%) & $53(35)$ & $162(59)$ & $<.0001$ \\
\hline Glomerular filtration rate, $\mathrm{mL} / \mathrm{min}$, mean \pm standard deviation ${ }^{a}$ & $31 \pm 11$ & $31 \pm 11$ & NS \\
\hline Urine sodium, $m E q / L$, mean \pm standard deviation & $26 \pm 24$ & $20 \pm 22$ & NS \\
\hline Urine volume, $m L / d$, mean \pm standard deviation & $1064 \pm 643$ & $817 \pm 690$ & .001 \\
\hline
\end{tabular}

NOTE. HCV, hepatitis $C$ virus.

${ }^{a}$ Estimated with the MDRD formula (Levey et alli1).

MELD score for the 3 or 4 different categories of renal failure is given in Figure 3. For all values of MELD score, the probability of death increased progressively from parenchymal renal failure to hypovolemia-related renal failure, renal failure associated with infections, and HRS.

\section{Discussion}

The current study reports the largest prospective investigation of renal failure in cirrhosis and provides information on the causes of renal failure in hospitalized cirrhotic patients and its relationship with prognosis. The most frequent causes of renal failure were infections

Table 3. Estimation of the Effect of the Cause of Renal Failure on 3-Month Mortality With Renal Failure Classified in 4 Categories: Parenchymal Nephropathy, Bacterial Infections, HypovolemiaRelated, and Hepatorenal Syndrome Defined With the Classical Diagnostic Criteria ${ }^{8}$

\begin{tabular}{lccc}
\hline & $\begin{array}{c}\text { Odds } \\
\text { ratio }\end{array}$ & $P$ & $\begin{array}{c}95 \% \text { confidence } \\
\text { interval }\end{array}$ \\
\hline Hypovolemia-related & 2.32 & .049 & $1.00-5.36$ \\
Bacterial infections & 2.61 & .027 & $1.11-6.11$ \\
Hepatorenal syndrome & 6.88 & .001 & $2.19-21.55$ \\
MELD score at diagnosis & 1.13 & $<.0005$ & $1.08-1.18$ \\
Serum sodium at & 0.96 & .020 & $0.92-0.99$ \\
$\quad$ diagnosis & & & $1.22-3.09$ \\
$\quad \begin{array}{l}\text { Hepatic encephalopathy } \\
\quad \text { at diagnosis }\end{array}$ & 1.94 & .005 & \\
\hline
\end{tabular}

NOTE. Reference category of final logistic model included parenchymal nephropathy. Potentially confounding variables evaluated in the model were MELD score, serum sodium, hepatic encephalopathy, mean arterial pressure, heart rate, prothrombin time, international normalized ratio, serum albumin, serum bilirubin, ascites, Child-Pugh score, and shock.

aGrades I to IV. followed by conditions associated with volume depletion, both accounting for almost two-thirds of all cases. Infections are a well-documented cause of renal failure in cirrhosis. ${ }^{1-3,12-14}$ In most cases, renal failure in patients with infections occurs in the absence of septic shock, as shown by the data of the current study. The pathogenic mechanism of renal failure in patients with cirrhosis and bacterial infections is not completely understood, but it is thought to be related to an impairment of the systemic arterial vasodilation present in cirrhosis because of bac-

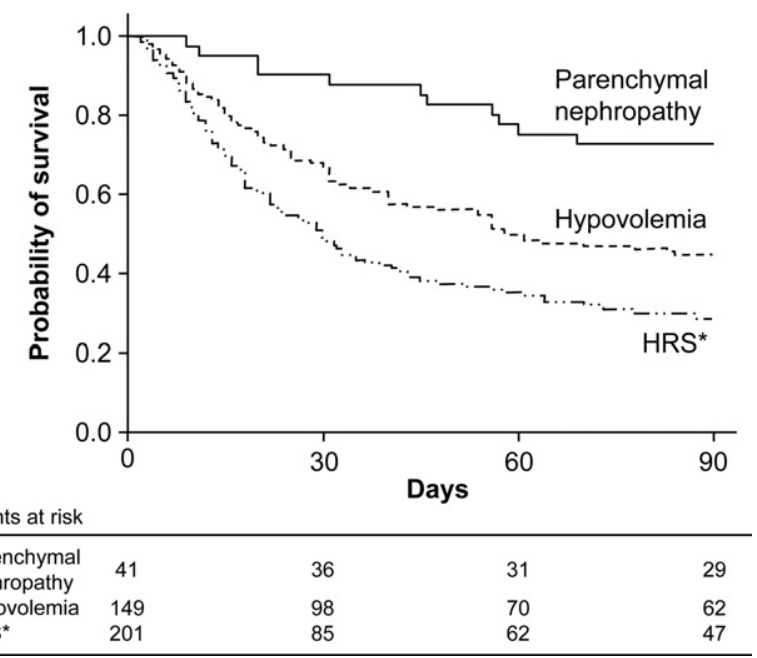

Figure 2. Three-month probability of survival of patients with cirrhosis and renal failure categorized in 3 different groups according to the cause of renal failure: renal failure due to parenchymal nephropathy, hypovolemia-associated renal failure, and HRS $(P<.0005)$. HRS was defined according to the new diagnostic criteria (Salerno et al9), which include patients with HRS diagnosed with the classical criteria (Arroyo et $\left.a^{8}\right)$ plus patients with active infections but without septic shock. Figures under the curves are patients at risk at different time points. 
Table 4. Estimation of the Effect of the Cause of Renal Failure on 3-Month Mortality With Renal Failure Classified in 3 Categories: Parenchymal Nephropathy, Hypovolemia-Related, and Hepatorenal Syndrome Defined With the Most Recent Diagnostic Criteria ${ }^{9}$

\begin{tabular}{lccc}
\hline & $\begin{array}{c}\text { Odds } \\
\text { ratio }\end{array}$ & $P$ & $\begin{array}{c}95 \% \text { confidence } \\
\text { interval }\end{array}$ \\
\hline Hypovolemia-related & 2.62 & .022 & $1.15-5.98$ \\
Hepatorenal syndrome & 3.48 & .004 & $1.48-8.17$ \\
MELD score at diagnosis & 1.14 & $<.0005$ & $1.09-1.19$ \\
Serum sodium at diagnosis & 0.96 & .037 & $0.93-0.99$ \\
\hline
\end{tabular}

NOTE. Reference category of final logistic model included parenchymal nephropathy. Potentially confounding variables evaluated in the model were MELD score, serum sodium, hepatic encephalopathy, mean arterial pressure, heart rate, prothrombin time, international normalized ratio, serum albumin, serum bilirubin, ascites, Child-Pugh score, and shock.

terial products or vasoactive mediators synthesized in relation with the infection. , $2,6,7,12-14$ Our results indicate that almost any bacterial infection may cause renal failure in cirrhosis, yet the most frequent are spontaneous infections (ie, infections caused by the spontaneous passage of bacteria mostly from the gastrointestinal tract to the systemic circulation), including spontaneous bacterial peritonitis and spontaneous bacteremia, followed by respiratory and urinary tract infections. It is important to note that, among patients with positive bacteriologic cultures, gram-positive bacteria were more common than gram-negative bacteria. These data are in agreement with the increasingly recognized importance of gram-positive bacteria as a cause of infection in hospitalized patients with cirrhosis. ${ }^{15,16}$ Fungal infections were also identified as a cause of renal failure, although their frequency was low.

Hypovolemia-related renal failure was the second cause of renal failure in the current series. The most common causes of hypovolemia were gastrointestinal bleeding followed by diuretics, which accounted for almost $75 \%$ of cases of renal failure related to hypovolemia. Most patients with hypovolemia developed renal failure in the absence of hypovolemic shock, which was present in only $17 \%$ of patients. HRS, as defined with the classical definition which excludes patients with active infections, ${ }^{8}$ was the third cause of renal failure, accounting for only $11 \%$ of cases of renal failure. However, if the new definition of HRS was used, ${ }^{9}$ the frequency of HRS increased up to $43 \%$, being the most frequent cause of renal failure in hospitalized patients. Parenchymal nephropathy was found to be a significant cause of renal failure in the current series. These data are in agreement with recent reports that emphasize the importance of intrinsic renal diseases in patients with cirrhosis. ${ }^{17}$ It is possible that the frequency of parenchymal nephropathy as a cause of renal failure could have been underemphasized because it was diagnosed only in patients with marked urine or ultrasonographic abnormalities, as suggested by internationally accepted criteria. ${ }^{8,9}$ Finally, it is worth mentioning that, although NSAIDs were described as a cause of renal failure almost 30 years ago ${ }^{18}$ and the use of these drugs in patients with cirrhosis is discouraged in all current practice guidelines, ${ }^{19-21}$ renal failure due to the administration of these drugs is still observed as a cause of admission to hospital (5.7\% in the current series).

The main finding of the current study is that the categorization of patients with cirrhosis and renal failure in 4 different groups with the use of a simple classification that is based on the cause of renal failure (renal failure associated with infections, hypovolemia-related renal failure, HRS, and parenchymal nephropathy) has prognostic relevance. This classification is based on clinical and analytical data and can be used in all settings. Patients with the best survival are those with renal failure due to parenchymal nephropathy (3-month probability of survival of $73 \%$ ), followed by patients with hypovolemia-related renal failure, patients with infection-associated renal failure, and patients with HRS (3-month prob-
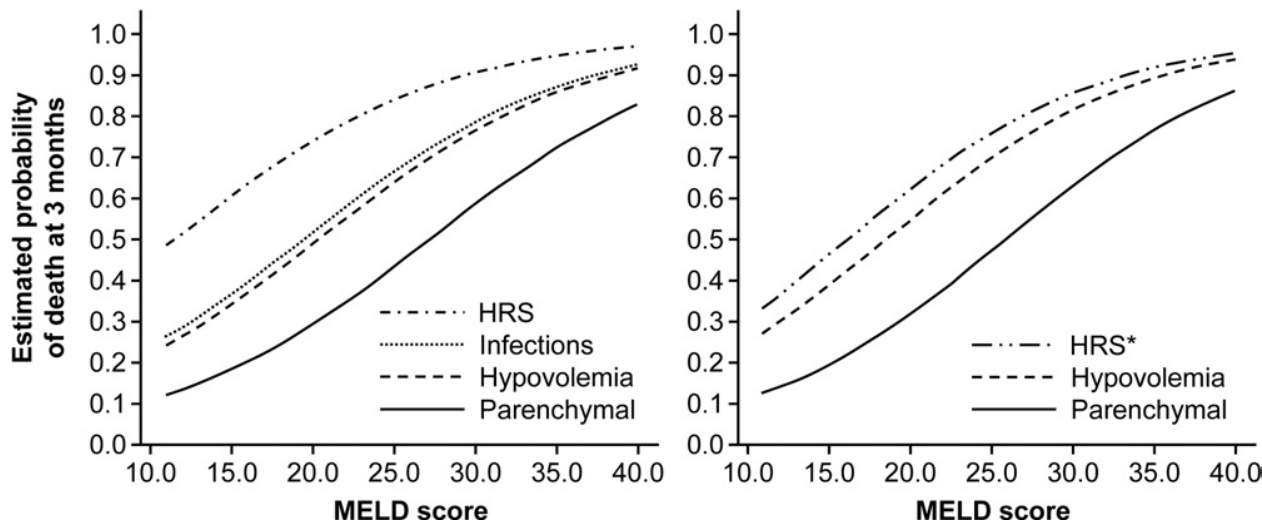

Figure 3. Plots of relationship of MELD score and 3-month probability of death in the different categories of renal failure. (Left graph) MELD scores according to 4 categories of renal failure as shown in Supplementary Table 4 (patients without hepatic encephalopathy). (Right graph) MELD scores according to 3 categories of renal failure, as shown in Supplementary Table 6. HRS, hepatorenal syndrome. ${ }^{*} H e p a t o r e n a l$ syndrome defined according to the most recent diagnostic criteria. ${ }^{9}$ 
ability of survival of $46 \%, 31 \%$, and $15 \%$, respectively). The most probable explanation for the good prognosis of patients with renal failure due to parenchymal renal diseases is that most of these patients had renal failure in the setting of a remarkably preserved liver and circulatory function, compared with the other groups of patients. On the basis of these findings, it is appealing to speculate that in patients with parenchymal renal diseases the development of renal failure, by increasing renal sodium retention, triggers the decompensation of cirrhosis and formation of ascites at a relatively early stage in the evolution of the liver disease. The relatively good prognosis of renal failure due to parenchymal nephropathy should be taken into account, particularly in patients who are candidates for transplantation in whom combined liverkidney transplantation is considered. Although the number of patients with cirrhosis and renal failure due to parenchymal nephropathy included in the current series was relatively small, it may be suggested that liver transplantation should not be offered to all patients with cirrhosis and renal failure due to parenchymal nephropathy, but only to those that in addition to renal failure have other poor prognostic indicators, including severe liver failure, low serum sodium concentration, or hepatic encephalopathy. This strategy, which should ideally be assessed in future specific studies, would allow for the reduction of the number of combined liver-kidney transplantations performed that has increased markedly since the introduction of the MELD score as the method for organ allocation in liver transplantation. ${ }^{22}$

Compared with patients with renal failure due to parenchymal nephropathy, patients with hypovolemia-related renal failure, renal failure associated with infections, and HRS had a much worse prognosis. This suggests that in cirrhosis, renal failure of hemodynamic origin (either because of loss of fluid from the intravascular compartment or because of arterial vasodilatation without fluid loss) has a greater negative effect on survival than renal failure of intrinsic renal origin. Moreover, these 3 groups of patients had marked liver failure which could have also contributed to their poor prognosis. The prognosis was remarkably dismal in patients with HRS and renal failure-associated with infections (3-month probability of survival of $15 \%$ and $31 \%$, respectively). The poor prognosis observed in patients with HRS is probably because almost two-thirds of patients had type 1 HRS, the type of HRS with the worst survival. ${ }^{1,8,9}$ A key finding of this study was the low survival rate of patients with renal failure associated with infection. This poor prognosis has also been reported in other studies, including a much smaller number of patients. ${ }^{6,13,14}$ Even if patients with septic shock were excluded from the group of patients with infection-associated renal failure, the 3 -month probability of survival remained remarkably low ( $24 \%$ vs $35 \%$, including and excluding patients with septic shock, respectively). When the group of patients with renal failure associated with infection without septic shock was combined with those with HRS, the 3-month probability of survival was only $29 \%$. These data clearly indicate that the research on renal failure in cirrhosis in the next years should be focused on mechanisms and prevention of renal failure in patients with infections with the objective of improving survival.

With the use of a logistic regression model, MELD score, serum sodium, and hepatic encephalopathy at diagnosis of renal failure together with the cause of renal failure were associated with prognosis. The finding that MELD score is a prognostic factor in patients with cirrhosis and renal failure has also been reported in several smaller series of patients with different types of renal failure. ${ }^{13,14,23}$ Nevertheless, it is important to point out that the specific prognostic implications of a given MELD score may vary substantially in different settings. ${ }^{24-26}$ A number of investigations have shown that serum sodium is an independent predictive factor of survival in patients with cirrhosis awaiting liver transplantation. ${ }^{24,25,27}$ Our study extends these observations by showing that serum sodium has also prognostic value in hospitalized patients with renal failure. The consistent findings of the prognostic value of serum sodium concentration across different populations of patients with cirrhosis and its independent value from other prognostic factors suggests that low serum sodium concentration per se has a negative effect on the natural history of cirrhosis. The relationship between low serum sodium and poor outcome requires investigation in future studies. Finally, the presence of hepatic encephalopathy at diagnosis was also an independent predictive factor of survival, emphasizing the relevance of hepatic encephalopathy in the outcome of patients with cirrhosis.

A number of other issues of this study also warrant discussion. First, the categorization of patients with renal failure used in the current study did not allow for the classification of $17.6 \%$ of patients with renal failure because they had a combination of several causes of renal failure or miscellaneous conditions. Unfortunately, currently no specific markers help in the identification of the cause of renal failure in cirrhosis. An effort should be made in the detection of markers of renal failure that may be useful for the differential diagnosis. Second, it could be argued that the cutoff value of serum creatinine used $(1.5 \mathrm{mg} / \mathrm{dL})$ was too high and that a lower value should have been used. This value was selected because it is widely recognized as the cutoff value of renal failure in cirrhosis and has been used in most published studies on renal failure in cirrhosis. ${ }^{1,8,9,12-14}$ Finally, it is important to emphasize that the results of the current study are only applicable to the population of hospitalized patients with cirrhosis and may not apply to ambulatory patients with cirrhosis and renal failure.

In conclusion, the results of this large prospective study indicate that in hospitalized patients with cirrhosis, infections are the main cause of renal failure, fol- 
lowed by hypovolemia-related renal failure, HRS, and parenchymal nephropathy. This classification in 4 categories has prognostic implications, with renal failure due to parenchymal nephropathy having the best prognosis and renal failure associated with infections and HRS the worst. MELD score, serum sodium, and hepatic encephalopathy add to the prognostic value of this classification of renal failure. This classification may be useful in the assessment of prognosis and may help in the decision making in liver transplantation.

\section{Supplementary Material}

Note: To access the supplementary material accompanying this article, visit the online version of Gastroenterology at www.gastrojournal.org, and at doi: $10.1053 /$ j.gastro.2010.07.043.

\section{References}

1. Ginès $P$, Schrier RW. Renal failure in cirrhosis. N Engl J Med 2009; 24;361:1279-1290.

2. Moreau R, Lebrec D. Acute renal failure in patients with cirrhosis: perspectives in the age of MELD. Hepatology 2003;37:233-243.

3. Garcia-Tsao G, Parikh CR, Viola A. Acute kidney injury in cirrhosis. Hepatology 2008;46:2064-2077.

4. Kamath P, Kim WR, and Advanced Liver Disease Group. The model for endstage liver disease. Hepatology 2007;45:797-805.

5. Terra C, Guevara M, Torre A, et al. Renal failure in patients with cirrhosis and sepsis unrelated to spontaneous bacterial peritonitis: value of MELD score. Gatroenterology 2005;129:19441953.

6. Ginès $P$, Cardenas $A$, Arroyo $V$, et al. Management of cirrhosis and ascites. N Engl J Med 2004;350:1646-1654.

7. Sort P, Navasa M, Arroyo V, et al. Effect of intravenous albumin on renal impairment and mortality in patients with cirrhosis and spontaneous bacterial peritonitis. N Engl J Med 1999;341:403-409.

8. Arroyo V, Ginès P, Gerbes AL, et al. Definition and diagnostic criteria of refractory ascites and hepatorenal syndrome in cirrhosis. International Ascites Club. Hepatology 1996;23:164-176.

9. Salerno F, Gerbes A, Ginès $P$, et al. Diagnosis, prevention and treatment of hepatorenal syndrome in cirrhosis. Gut 2007;56: 1310-1318.

10. Rimola A, Garcia-Tsao G, Navasa M, et al. Diagnosis, treatment, and prophylaxis of spontaneous bacterial peritonitis: a consensus document of the International Ascites Club. J Hepatol 2000; 32:142-153.

11. Levey S, Greene T, Kusek JW, et al. A simplified equation to predict glomerular filtration rate from serum creatinine. J Am Soc Nephrol 2000;11:155A.

12. Follo A, Llovet JM, Navasa M, et al. Renal impairment after spontaneous bacterial peritonitis in cirrhosis: incidence, clinical course, predictive factors and prognosis. Hepatology 1994;20: 1495-1501.

13. Fasolato $S$, Angeli $P$, Dallagnese $L$, et al. Renal failure and bacterial infections in patients with cirrhosis: epidemiology and clinical features. Hepatology 2007;45:223-229.

14. Thabut D, Massard J, Gangloff A, et al. Model for end-stage liver disease score and systemic inflammatory response are major prognostic factors in patients with cirrhosis and acute functional renal failure. Hepatology 2007;46:1872-1882.

15. Fernández J, Navasa M, Gómez J, et al. Bacterial infections in cirrhosis: epidemiological changes with invasive procedures and norfloxacin prophylaxis. Hepatology 2002;35:140-148.
16. Campillo B, Richardet JP, Kheo T, et al. Nosocomial spontaneous bacterial peritonitis and bacteremia in cirrhotic patients: impact of isolate type on prognosis and characteristics of infection. Clin Infect Dis 2002;35:1-10.

17. Trawalé JM, Paradis V, Rautou PE, et al. The spectrum of renal lesions in patients with cirrhosis: a clinicopathological study. Liver Int 2010;30:725-32.

18. Boyer TD, Zia P, Reynolds TB. Effect of indomethacin and prostaglandin A1 on renal function and plasma renin activity in alcoholic liver disease. Gastroenterology 1979;77:215-222.

19. Moore KP, Wong F, Ginès $P$, et al. The management of ascites in cirrhosis: report on the consensus conference of the International Ascites Club. Hepatology 2003;38:258-266.

20. Runyon BA. Management of adult patients with ascites due to cirrhosis: an update. AASLD Practice Guidelines Committee. Hepatology 2009;49:2087-2107.

21. Garcia-Tsao G, Lim JK; Members of Veterans Affairs Hepatitis C Resource Center Program. Management and treatment of patients with cirrhosis and portal hypertension: recommendations from the Department of Veterans Affairs Hepatitis C Resource Center Program and the National Hepatitis C Program. Am J Gastroenterol 2009;104:1802-1829.

22. Charlton MR, Wall WJ, Ojo AO, et al. Report of the first international liver transplantation society expert panel consensus conference on renal insufficiency in liver transplantation. International Liver Transplantation Society Expert Panel. Liver Transpl 2009;15:S1-34.

23. Alessandria $C$, Ozdogan $O$, Guevara $M$, et al. MELD score and clinical type predict prognosis in hepatorenal syndrome: relevance to liver transplantation. Hepatology 2005;41:1282-1289.

24. Heuman DM, Abou-Assi SG, Habib A, et al. Persistent ascites and low serum sodium identify patients with cirrhosis and low MELD scores who are at high risk for early death. Hepatology 2004;40: 802-810.

25. Kim WR, Biggins SW, Kremers WK, et al. Hyponatremia and mortality among patients on the liver-transplant waiting list. N Engl J Med 2008;359:1018-1026.

26. Stewart CA, Malinhoc M, Kim WR, Kamath PS. Hepatic encephalopathy as a predictor of survival in patients with end-stage liver disease. Liver Transpl 2007;13:1366-1371.

27. Londoño MC, Cárdenas A, Guevara M, et al. MELD score and serum sodium in the prediction of survival of patients with cirrhosis awaiting liver transplantation. Gut 2007;56:1283-1290.

Received March 2, 2010 Accepted July 22, 2010.

\section{Reprint requests}

Address requests for reprints to: Pere Ginès, MD, Liver Unit, Hospital Clínic, Villarroel, 170, 08036, Barcelona, Spain. e-mail: pgines@clinic.ub.es; fax: xxx.

\section{Acknowledgments}

The authors thank Raquel Cela, RN, and the nursing staff of the Liver Unit and Intensive Care Unit for their participation in the study.

\section{Conflicts of interest}

The authors disclose no conflicts.

\section{Funding}

This work was supported by a grant from Fondo de Investigación Sanitaria (FIS PI080126; to P.G.) and a grant from Premio Fundación Lilly de Investigación Biomédica Clínica (to J.R.). M.M.-L. and G.P. were supported by grants from the Fundación Banco de Bilbao-Vizcaya-Argentaria (FBBVA). C.F. was supported by a grant from Instituto Reina Sofía de Investigación Nefrológica. CIBEREHD is funded by the Instituto de Salud Carlos III. 


\section{Supplementary Materials and Methods}

\section{Causes of Renal Failure: Mixed Cause, Nephrotoxicity, and Miscellaneous Causes}

Of the 45 patients with mixed causes of renal failure ( $8 \%$ of the whole series of patients), the most common combination was infection and hypovolemia which occurred in 41 patients (91\%). The remaining 4 patients had combination of parenchymal nephropathy, infection, and hypovolemia (3 patients) and parenchymal nephropathy plus hypovolemia (1 patient). No patient had a combination of HRS with other causes of renal failure because by definition HRS could only be diagnosed when other causes of renal failure had been excluded.8,9

In 42 patients $(7.5 \%$ of the whole series) renal failure was considered secondary to nephrotoxicity because of the existence of a close chronologic relationship between the administration of a potentially nephrotoxic agent and renal failure. In 19 of the 42 patients no cause of renal failure other than the administration of a potentially nephrotoxic agent could be identified (NSAIDs in 14 patients, antibiotics in 3 , and $\beta$-blockers and vasodilators in 1 patient each). In the remaining 23 patients, there was a coexistence of administration of a potentially nephrotoxic agent (NSAIDs in 18 patients and antibiotics, $\beta$-blockers, vasodilators, contrast media, and amphotericin B in 1 patient each) and another potential cause of renal failure (hypovolemia and infection in 13 and 15 patients, respectively). In total, the administration of
NSAIDs was involved in $5.7 \%$ of patients in the whole series (32 of the 562 patients) and in $76.2 \%$ (32 of 42) of patients with nephrotoxicity as the cause of renal failure.

In 12 patients $(2.1 \%$ of the whole series) renal failure was secondary to miscellaneous conditions (severe cardiac failure in 8 patients, obstructive nephropathy in 3 patients, and rhabdomyolysis in 1 patient).

The survival of these 3 subsets of patients is shown in Supplementary Figure 1. Patients with nephrotoxicity or miscellaneous conditions had a 3-month probability of survival significantly higher than patients with mixed causes of renal failure. The 3-month probability of survival of patients with mixed causes of renal failure was similar to that of the 463 patients included in the 4 specific categories of renal failure described in the body of the article (33\% vs $38 \%$, respectively; $P=\mathrm{NS}$ ).

\section{Probability of Death According to MELD Score for Each Category of Renal Failure}

To analyze the relationship between MELD score and survival for each category of renal failure, prognostic formulae were constructed so that the importance of the cause of renal failure in terms of MELD points could be assessed. The prognostic formula used for the calculation of survival has been described in "Materials and Methods." Specific prognostic formulae used for each category of renal failure are shown in Supplementary Tables 2 and 3. The relationship between the probability of death and MELD score within each category of renal failure are shown in Supplementary Tables 4-6. 

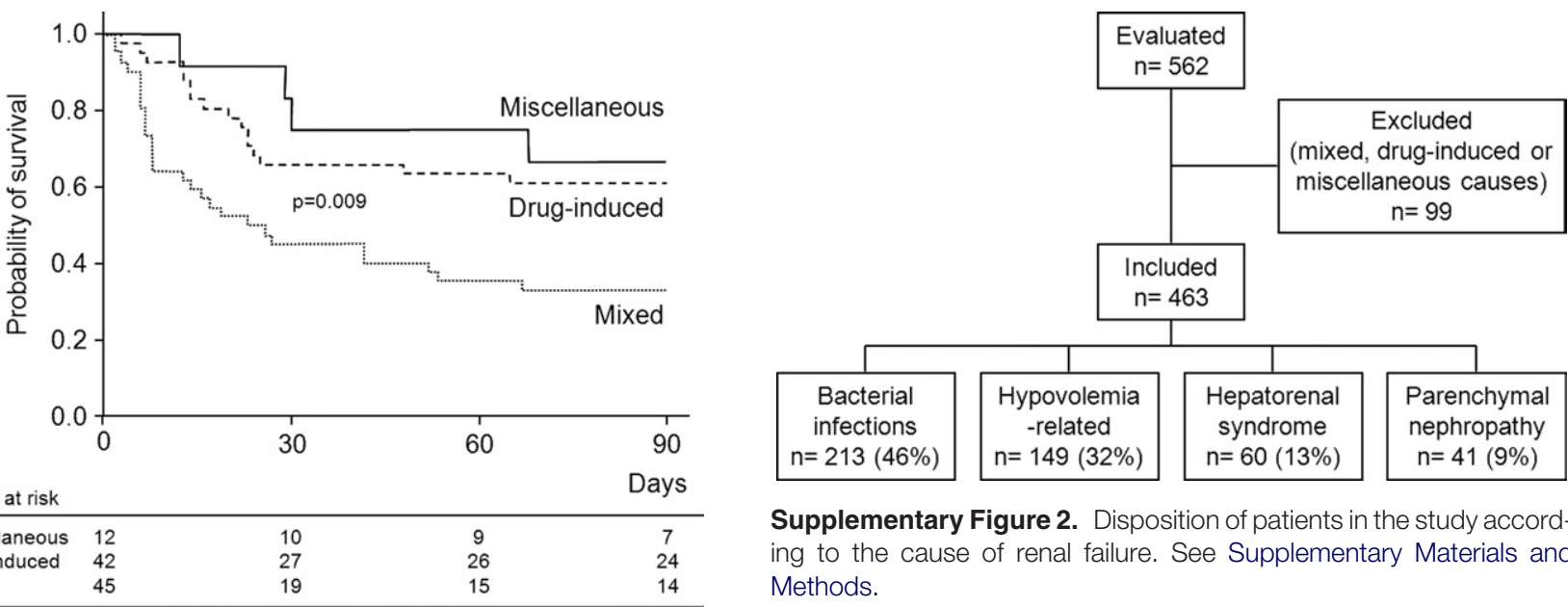

\begin{tabular}{lllcc} 
Patients at risk & \multicolumn{1}{l}{} \\
\hline - Miscellaneous & 12 & 10 & 9 & 7 \\
- Drug-induced & 42 & 27 & 26 & 24 \\
- Mixed & 45 & 19 & 15 & 14 \\
\hline
\end{tabular}

Supplementary Figure 2. Disposition of patients in the study according to the cause of renal failure. See Supplementary Materials and Methods.

Supplementary Figure 1. Three-month probability of survival of patients with cirrhosis and renal failure due to mixed causes of renal failure (combination of infection, hypovolemia, or parenchymal nephropathy; 45 patients), drug-induced (42 patients), and miscellaneous (12 patients) conditions. Figures under the curves are patients at risk at different time points. 


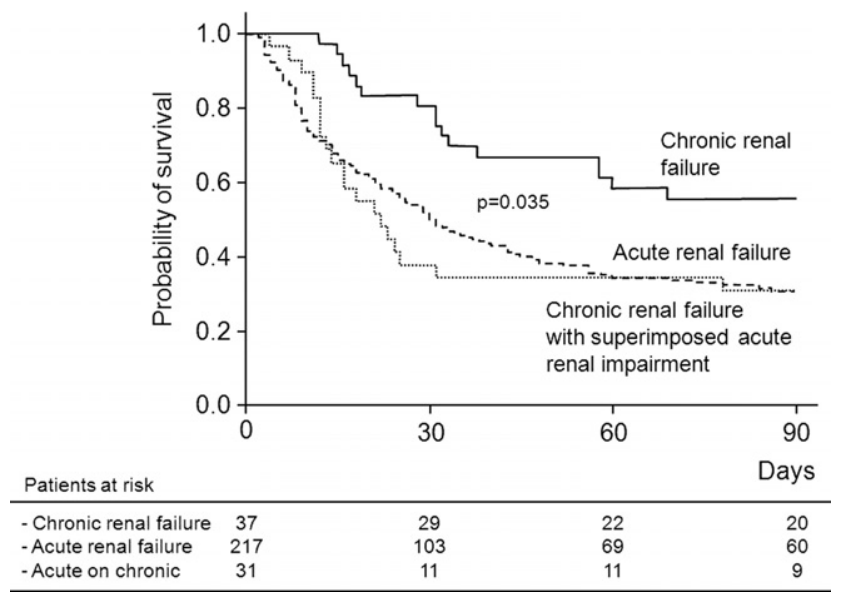

Supplementary Figure 3. Three-month probability of survival of 285 patients with an available serum creatinine concentration within 3 months before admission divided into 3 groups: chronic renal failure, chronic renal failure with superimposed acute renal impairment, and acute renal failure. Figures under the curves are patients at risk at different time points.
Supplementary Table 2. Prognostic Formulae Used in the Calculation of the Probability of Death According to MELD Score in the Model With 4 Categories

\begin{tabular}{ll}
\hline Category of Renal Failure & Logit $^{a}$ \\
\hline Infection-associated & MELD $\times 0.123-2.374$ \\
Without encephalopathy & MELD $\times 0.123-1.712$ \\
$\quad$ With encephalopathy & \\
Hypovolemia-related & MELD $\times 0.123-2.491$ \\
Without encephalopathy & MELD $\times 0.123-1.829$ \\
$\quad$ With encephalopathy & MELD $\times 0.123-1.403$ \\
Hepatorenal syndrome & MELD $\times 0.123-0.741$ \\
$\quad$ Without encephalopathy & \\
With encephalopathy & MELD $\times 0.123-3.332$ \\
Parenchymal nephropathy & Mithout encephalopathy \\
With encephalopathy & MELD $\times 0.123-2.670$ \\
\hline
\end{tabular}

NOTE. Serum sodium was set at $130 \mathrm{mEq} / \mathrm{L}$ (median value in the whole series).

${ }^{\text {aLogit }}=1.998+$ MELD $\times 0.123-$ serum sodium $\times 0.041+$ hepatic encephalopathy at diagnosis $\times 0.662+$ hypovolemia-related $\times$ $0.841+$ bacterial infections $\times 0.958+$ hepatorenal syndrome $\times$ 1.929 .

Supplementary Table 1. Distribution of Patients of the 4 Different Categories of Renal Failure According to the Status of Renal Function Before Hospitalization

\begin{tabular}{|c|c|c|c|}
\hline & $\begin{array}{c}\text { No previous renal failure, } \\
\mathrm{n}(\%)\end{array}$ & $\begin{array}{c}\text { Chronic renal failure, } \\
\text { n (\%) }\end{array}$ & $\begin{array}{l}\text { Chronic renal failure with } \\
\text { superimposed acute } \\
\text { renal impairment, } \mathrm{n}(\%)\end{array}$ \\
\hline Infection associated renal failure $(\mathrm{n}=129)^{a}$ & $112(87)$ & $8(6)$ & $9(7)$ \\
\hline Hypovolema-related renal failure $(n=108)^{a}$ & $80(74)$ & $17(16)$ & $11(10)$ \\
\hline Hepatorenal syndrome $(n=36)^{a}$ & $24(67)$ & 5 (14) & 7 (19) \\
\hline Parenchymal nephropathy $(n=12)^{a}$ & $1(8)$ & $7(58)$ & $4(33)$ \\
\hline
\end{tabular}

The number of patients with serum creatinine values available within a period of 3 months before hospitalization. 
Supplementary Table 3. Prognostic Formulae Used in the Calculation of the Probability of Death According to MELD Score in the Model With 3 Categories

\begin{tabular}{lc}
\hline Category of renal failure & Logit \\
\hline Hypovolemia-related & MELD $\times 0.131-2.425$ \\
Hepatorenal syndrome & MELD $\times 0.131-2.143$ \\
Parenchymal nephropathy & MELD $\times 0.131-3.389$ \\
\hline
\end{tabular}

NOTE. Serum sodium was set at $130 \mathrm{mEq} / \mathrm{L}$, the median value in the whole series.

Logit $=1.881+$ MELD $\times 0.131-$ serum sodium $\times 0.040+$ hypovolemia-related $\times 0.964+$ hepatorenal syndrome $\times 1.246$.

Supplementary Table 4. MELD Values Corresponding to the Different Levels of Probability of Death in Patients Without Hepatic Encephalopathy Classified Into 4 Different Categories of Renal Failure

\begin{tabular}{ccccc}
\hline $\begin{array}{c}\text { Probability of death } \\
\text { at } 3 \text { mo }\end{array}$ & $\begin{array}{c}\text { Parenchymal nephropathy, } \\
\text { MELD score }\end{array}$ & $\begin{array}{c}\text { Hypovolemia-related, } \\
\text { MELD score }\end{array}$ & $\begin{array}{c}\text { Infection-associated, } \\
\text { MELD score }\end{array}$ & $\begin{array}{c}\text { Hepatorenal syndrome, } \\
\text { MELD score }\end{array}$ \\
\hline 0.9 & 45.0 & 38.2 & 37.2 & 29.4 \\
0.8 & 38.4 & 31.6 & 30.7 & 22.8 \\
0.7 & 34.1 & 27.2 & 22.3 & 18.4 \\
0.6 & 30.5 & 23.6 & 19.4 & 14.8 \\
0.5 & 27.2 & 20.3 & 16.1 & 1.5 \\
0.4 & 23.9 & 17.0 & 12.5 & 4.2 \\
0.3 & 20.3 & 13.4 & 8.1 & 0.2 \\
\hline .2 & 15.9 & 9.1 & & 0 \\
\hline
\end{tabular}

NOTE. Serum sodium was set at $130 \mathrm{mEq} / \mathrm{L}$, the median value in the whole series.

Supplementary Table 5. MELD Values Corresponding to the Different Levels of Probability of Death in Patients With Hepatic Encephalopathy Classified Into 4 Different Categories of Renal Failure

\begin{tabular}{ccccc}
\hline $\begin{array}{c}\text { Probability of death } \\
\text { at } 3 \text { months }\end{array}$ & $\begin{array}{c}\text { Parenchymal nephropathy, } \\
\text { MELD score }\end{array}$ & $\begin{array}{c}\text { Hypovolemia-related, } \\
\text { MELD score }\end{array}$ & $\begin{array}{c}\text { Infection-associated, } \\
\text { MELD score }\end{array}$ & $\begin{array}{c}\text { Hepatorenal syndrome, } \\
\text { MELD score }\end{array}$ \\
\hline 0.9 & 39.7 & 30.8 & 31.9 & 24.0 \\
0.8 & 33.1 & 24.6 & 25.3 & 17.4 \\
0.7 & 28.7 & 20.5 & 20.9 & 17.3 \\
0.6 & 25.1 & 17.1 & 14.0 & 9.4 \\
0.5 & 21.8 & 14.0 & 10.7 & 6.1 \\
0.4 & 18.5 & 10.9 & 7.1 & 2.8 \\
0.3 & 14.9 & 7.6 & 2.7 & - \\
0.2 & 10.5 & 3.5 & & - \\
\hline
\end{tabular}

NOTE. Serum sodium was set at $130 \mathrm{mEq} / \mathrm{L}$, the median value in the whole series.

Supplementary Table 6. MELD Values Corresponding to the Different Levels of Probability of Death in Patients Classified Into 3 Different Categories of Renal Failure

\begin{tabular}{cccr}
\hline $\begin{array}{c}\text { Probability of death } \\
\text { at } 3 \text { mo }\end{array}$ & $\begin{array}{c}\text { Parenchymal nephropathy, } \\
\text { MELD score }\end{array}$ & $\begin{array}{c}\text { Hypovolemia-related, } \\
\text { MELD score }\end{array}$ & $\begin{array}{c}\text { Hepatorenal syndrome, } \\
\text { MELD score }\end{array}$ \\
\hline 0.9 & 42.6 & 35.3 & 33.1 \\
0.8 & 36.5 & 29.1 & 26.9 \\
0.7 & 32.3 & 25.0 & 22.8 \\
0.6 & 29.0 & 21.6 & 19.5 \\
0.5 & 25.9 & 18.5 & 16.4 \\
0.4 & 22.8 & 15.4 & 13.3 \\
0.3 & 19.4 & 12.0 & 9.9 \\
0.2 & 15.3 & 7.9 & 5.8 \\
\hline
\end{tabular}

NOTE. Serum sodium was set at $130 \mathrm{mEq} / \mathrm{L}$, the median value in the whole series.

${ }^{a}$ According to the new diagnostic criteria (Salerno et $\mathrm{al}^{9}$ ). 